\title{
Construction of a densely poly(ethylene glycol)- chain-tethered surface and its performance
}

\author{
Yukio Nagasaki ${ }^{1,2,3}$
}

Non-biofouling surfaces constitute one of the most important subjects in sensitively and selectively detecting biomolecular events. Poly(ethylene glycol) (PEG) chains tethered on substrate surfaces are well known to reduce non-biofouling characteristics. Protein adsorption onto a PEG-chain-tethered surface is strongly influenced by the density of the PEG chain and is almost completely suppressed by the successive treatment of longer PEG chains (5 kDa) followed by the treatment of PEG ( $2 \mathrm{kDa}$; mixed-PEG-chain-tethered surface) because of a significant increase in PEG chain density. To modify versatile substrate surface, PEG possessing pentaethylenehexamine at one end (N6-PEG) was prepared via a reductive amination reaction of aldehyde-ended PEG with pentaethylenehexamine. Using N6-PEG, antibody/PEG co-immobilization was conducted on a substrate possessing active ester groups. After the antibody was immobilized on the surface, PEG tethered chains were constructed surrounding the immobilized antibody. It is interesting to note that the PEG-chain-tethered functions not only as a non-fouling agent but also improves immune response. The hybrid surface was also applied to oligo DNA immobilization. The oligo DNA/PEG hybrid surface improved hybridization, retaining its non-fouling ability. Densely packed PEG tethered chains surrounding antibodies and/or oligo DNA improved their orientation on the surface. Thus, this material is promising as a high-performance biointerface for versatile applications.

Polymer Journal (2011) 43, 949-958; doi:10.1038/pj.2011.93; published online 5 October 2011

Keywords: antibody; biosensing; DNA; end-functionalized poly(ethylene glycol); enzyme-linked immunosorbent assay; hybrid biointerface; soft interface

\section{INTRODUCTION}

Specific biorecognition on substrate surfaces has long been utilized in many applications such as biosensing, ${ }^{1}$ immunodiagnostics, ${ }^{2}$ enzyme immunoassay, ${ }^{3}$ western blotting ${ }^{4}$ and protein microarrays. ${ }^{5}$ Important factors for the improvement in specific biorecognition on surfaces are the suppression of nonspecific biofouling and the suitable orientation of immobilized biomolecules. The former decreases nonspecific noise and the background level, and the latter increases the specific recognition level. To improve the non-biofouling character of surfaces, numerous efforts have been undertaken. In general, natural polymers such as albumin, casein and dextran have been used for blocking on substrate surfaces. ${ }^{6}$ These treatments work well and suppress protein biofouling extensively. If extremely high performance is required, however, these blocking treatments are not enough. In addition, natural products, especially those derived from animals, may have undesired contents such as bacteria and viruses, which may affect the user. ${ }^{7}$ Under these circumstances, synthetic polymers have been recently suggested as suitable surface blocking agents. Numerous types of water-soluble polymers, such as poly(acrylamide), poly(vinyl alcohol), poly(vinyl pyrrolidone) and poly(ethylene glycol) (PEG), have been investigated so far. Recently, new types of synthetic polymers such as poly(methoxy acrylate) and poly(2-methacryloyloxyethyl phosphorylcholine) have been developed.

PEG is one of the most popular water-soluble polymers and is used as a surface-modification agent. Nagaoka and his co-workers ${ }^{8}$ first reported in 1982 that a 'PEG polymer brush,' which denotes one PEG chain-end immobilized on a substrate surface to form a tethered chain, improved anti-thrombogenicity. Since their report, many reports on PEG-chain-tethered surfaces have been published. ${ }^{9-12}$ We have begun to investigate the performance of PEG tethered chains in producing anti-biofouling effects using our original heterobifunctional PEG. From our investigation, both chain length and density of PEG are important factors in improving the non-biofouling character of surfaces. ${ }^{13}$ An increase in the PEG molecular weight (MW) results in a decrease in the chain density of the PEG layer due to the increased excluded-volume effect, which causes solute penetration into the PEG layer to induce nonspecific interactions with the substrate. Thus, the length and density of the tethered PEG chains produce a tradeoff in terms of the sensitivity and selectivity of biomolecule recognition. To overcome this tradeoff, a mixed-PEG-chain-tethered surface with a

${ }^{1}$ Department of Materials Sciences, Graduate School of Pure and Applied Sciences, University of Tsukuba, Tsukuba, Ibaraki, Japan; ${ }^{2}$ Master's School of Medical Sciences, Graduate School of Comprehensive Human Sciences, University of Tsukuba, Tsukuba, Ibaraki, Japan and ${ }^{3}$ Satellite Laboratory, International Center for Materials Nanoarchitectonics, National Institute for Materials Science, University of Tsukuba, Tsukuba, Ibaraki, Japan

Correspondence: Professor Y Nagasaki, Department of Materials Sciences, Graduate School of Pure and Applied Sciences, University of Tsukuba, Tennoudai 1-1-1, Tsukuba, Ibaraki 305-8573, Japan.

E-mail: yukio@nagalabo.jp

Received 29 June 2011; revised 5 August 2011; accepted 8 August 2011; published online 5 October 2011 
filler layer of short PEG ( $2 \mathrm{kDa}$ underlain on the pre-constructed longer PEG brush layer) was prepared. ${ }^{14}$ The surface composed of mixed-PEG tethered chains almost completely prevented the nonspecific adsorption of bovine serum albumin (BSA). The present review describes the effect of mixed PEG on the non-biofouling character and also hybrid construction of a PEG/biopolymer mixture on substrate surfaces for versatile applications. Because many excellent reviews on PEG surface coating have been previously published, ${ }^{15-28}$ this review mainly introduces our work.

\section{BACKGROUND}

PEG has long been manufactured industrially and utilized in many applications such as nonionic surfactants, lubricants, an intermediate for urethane composition, adhesives and cosmetics. ${ }^{29}$ PEG is non-toxic and is also used for bio-related applications such as pharmaceutical formulation, ${ }^{30}$ aqueous two-phase partition, ${ }^{31}$ precipitant for plasmid DNA isolation, ${ }^{32}$ protein crystallization ${ }^{33}$ and cell fusion. $^{34}$

Among water-soluble polymers, PEG has the following characteristics. ${ }^{35}$ PEG has a small $\chi$-parameter $(\chi<0.5)$, which is an index of the interaction between a polymer and solvent (Flory-Huggins interaction). This means that PEG is completely miscible in water, owing to the hydrogen bonding between water molecules and ether oxygen in the PEG chain. PEG has one of the largest second virial coefficients among water-soluble polymers, which means that it adopts a wellexpanded conformation in aqueous media. From Flory's dilute solution theory, the second virial coefficient is affected by the chain-chain excluded volume. The large second virial coefficient of PEG denotes a large excluded volume among water-soluble polymers. PEG also has a small mobility factor value, $\sigma$, which denotes the root-mean-square end-to-end distance $\left\langle R^{2}\right\rangle^{1 / 2}$ of the polymer concerned divided by that of an ideal freely rotating chain. Thus, a large $\sigma$-value indicates a polymer with a stiff rod-like chain in solution. The small $\sigma$-value of PEG means high mobility in aqueous media. Thus, PEG has (i) high water compatibility, (ii) a large exclusion volume and (iii) extremely high flexibility in aqueous media.

From these perspectives, PEG has begun to have an important role in the development of high-performance biointerfaces. This is especially true regarding the versatile techniques for surface coating using PEG that have been developed since the 1980s to improve blood and bio-compatibility. Figure 1 summarizes several methods for the immobilization of PEG on substrate surfaces: PEG gels, ${ }^{19,20,36}$ the physical and chemical immobilization of $\mathrm{PEG}^{13,21,37}$ (so-called grafting-to method), the adsorption of block $22-27,38$ and graft $^{27,28,39,40}$ copolymers, polymerization from the surface ${ }^{41}$ (the so-called grafting-from method), the immobilization of star-shape polymers and micelles, ${ }^{42-44}$ and other methods.

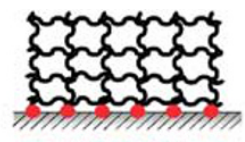

hydrogel

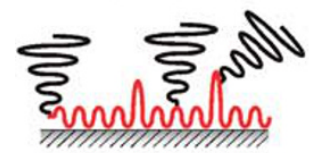

Graft Copolymer

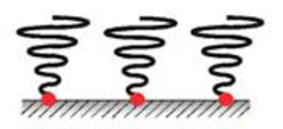

Graft To

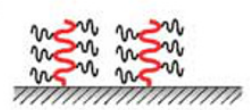

Graft from

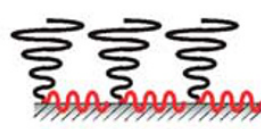

Block Copolymer

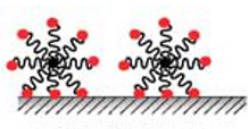

Star Polymer
Figure 1 Schematic illustrations of surface modifications achieved using poly(ethylene glycol) derivatives.

\section{CONSTRUCTION OF A DENSELY PEG-CHAIN-TETHERED SURFACE FOR HIGH ANTI-FOULING CHARACTER}

There are several methods used to construct PEG tethered chains on surfaces. Two main techniques are known as the 'grafting-from' and 'grafting-to' methods; the former is used to construct PEG chains by the polymerization of vinyl monomers from a surface, and the latter involves the immobilization of polymer to a surface. The graftingfrom technique has been thoroughly studied by Tsujii and his co-workers ${ }^{41}$ and is not described in this review. The grafting-to method is performed by inducing a specific interaction between the polymer and the surface, namely, hydrophobic interaction, electrostatic interaction or covalent conjugation. To suppress protein adsorption, a PEG-chain-tethered surface with different chain lengths was prepared by the adsorption of PEG- $b$-poly(dl-lactide) (PEG- $b$-PLA) onto a PLA surface by hydrophobic interactions.

Protein adsorption onto PEG- $b$-PLA surfaces was then measured using BSA as a model protein. Figure 2 shows the BSA adsorption from Dulbecco phosphate-buffered saline $(-)$ solution onto various PEG- $b$-PLA surfaces. On a PLA surface, BSA was significantly adsorbed, while on PEG-coated surfaces BSA adsorption clearly decreased. As the MW of the PEG chain was increased, the amount of BSA adsorbed onto the surface significantly decreased to a PEG MW of about 3300. A further increase in the PEG chain length resulted in a slight increase in BSA adsorption. The same trend was observed for the adsorption of BSA from both 0.01 and $4.5 \mathrm{mg} \mathrm{ml}^{-1}$ BSA solutions, although the amount of BSA adsorbed from the latter solution was almost twice the BSA adsorption from the former solution, in the region with shorter PEG chain lengths. The reduced adsorption of BSA was more pronounced in the region with higher PEG chain lengths, especially at the PEG/PLA (3.3/5.4) surface, which showed a minimum adsorption of BSA. The high MW of PEG, however, did not completely eliminate protein adsorption. There are several possible explanations for the decrease in protein adsorption

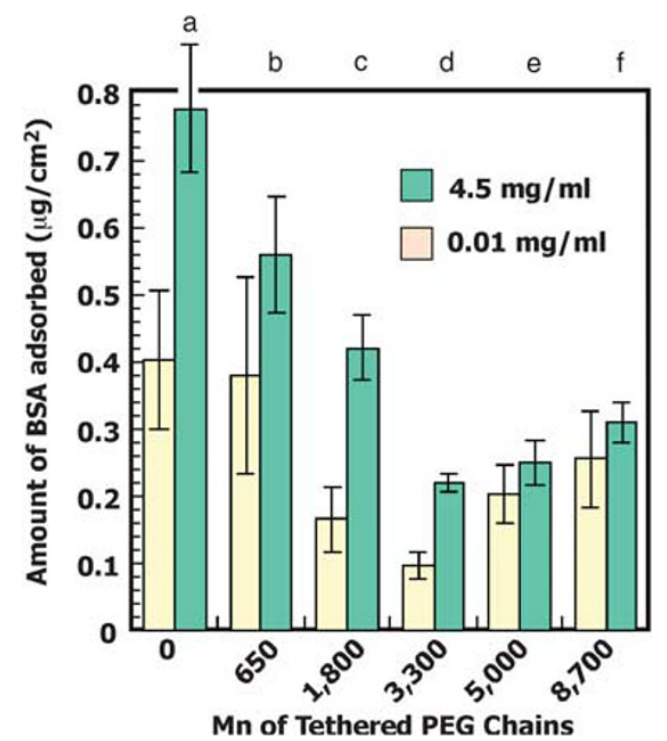

Figure 2 Adsorbed amount of bovine serum saline (BSA) on poly(dl-lactide) (PLA) and poly(ethylene glycol) (PEG)/PLA block copolymer surfaces at room temperature after incubation for $90 \mathrm{~min}$ : (a) PLA homopolymer and PEG- $b$ PLA block (PEG-b-poly(dl-lactide)) copolymers of different PEG- $b$-PLA segments; (b) PEG- $b$-PLA (0.65/11.5), (c) PEG- $b$-PLA (1.8/7.0), (d) PEG- $b$ PLA (3.3/5.4), (e) PEG-b-PLA (5.0/4.6), and (f) PEG-b-PLA (8.7/6.9). This figure is reproduced from the study by Hidenori et al., ${ }^{10}$ by courtesy of the publishers, American Chemical Society, USA. 
with the change in the MW of PEG, including a large excluded volume, osmotic repulsion, the high mobility of the PEG molecule, the high water content of PEG surfaces and the low interfacial free energy of PEG with water. Regardless of the mechanism, the increase in the thickness of the PEG surface layer is very important factor. However, an increase in the PEG chain length results in a decrease in the chain density of the PEG layer due to the increased excludedvolume effect, causing solute penetration into the PEG layer to induce nonspecific interactions with the substrate. Thus, the length and density of the tethered PEG chain constitute a tradeoff in terms of the sensitivity and selectivity of biomolecule recognition. To construct a surface with a highly selective biosensing capability through PEG modification, a tethered PEG layer is required to satisfy the creation of both long chain length and high density to ensure nonfouling.

With the increase in the MW of PEG tethered chains, the nonbiofouling character was observed to improve as stated above. Tethered chains that are too long, however, tend to enhance the nonspecific adsorption of proteins. How can we overcome this tradeoff relationship? To construct rather longer PEG chains while retaining high PEG chain density, a mixed-PEG-chain-tethered surface, which suggests the introduction of a short under-brush PEG layer to the surface pre-modified with comparatively long PEG chains, was constructed (Scheme 1).

Figure 3 shows the protein adsorption character of a surface plasmon resonance (SPR) sensor chip coated with sulfanyl-ended PEG (SH-PEG) tethered chains as a function of protein sizes. ${ }^{45}$ The mixed-PEG chain tethered on a gold surface was created by the successive immobilization of SH-PEG $(5 \mathrm{kDa})$ followed by SH-PEG $(2 \mathrm{kDa}) .{ }^{13}$ When dextran gel was used as a control, nonspecific adsorption was prevented to some extent when using high-molecular-weight protein. With the decreasing size of the protein, the nonspecific adsorption increased significantly. The conventional PEG-chain-tethered surface prepared with PEG (5kDa) suppressed the nonspecific adsorption of the proteins possessing a MW higher than $10 \mathrm{kDa}$. However, the performance was too low at MWs below $10 \mathrm{kDa}$. In the case of the mixed-PEG-chain-tethered surface, complete non-fouling was observed. In particular, the mixed PEG surface prevented not only the absorption of large MW proteins but also that of small proteins and peptides. Actually, even tetrapeptides (RGDS, $\mathrm{MW}=450$ ) could be rejected almost completely.

\section{SYNTHESIS OF END-REACTIVE PEG FOR SURFACE MODIFICATIONS}

PEG modification is widely applied in developing versatile substrate surfaces not only for PLA and gold surfaces as stated above, but also for various types of plastics, metals and metal oxides. We have recently

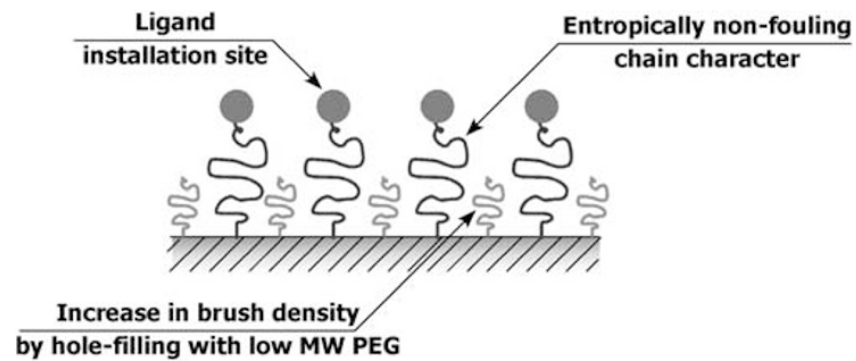

Scheme 1 Mixed-poly(ethylene glycol) (PEG) tethered-chain surface (long PEG chain improves non-fouling character, and short PEG chain increases PEG chain density). synthesized PEG possessing pentaethylenehexamine at one end (N6PEG; Scheme 2). N6-PEG can be applied not only to gold surfaces but also to other material surfaces; active-ester surfaces are particularly suitable.

For PEG modification on a gold surface, PEG-SH is commonly used, which has shown excellent inhibition of the adsorption of nonspecific molecules. ${ }^{46,47}$ However, the Au-S linkage is not strong enough under physiological conditions because of the limited oxidative stability of thiolate species, ${ }^{48-50}$ as well as the exchange reactions with thiolated compounds that occur inside the body. Thiolatemodified surfaces are also damaged by exposure to light, high temperature and oxygen. ${ }^{48-50}$ To improve the long-term stability of

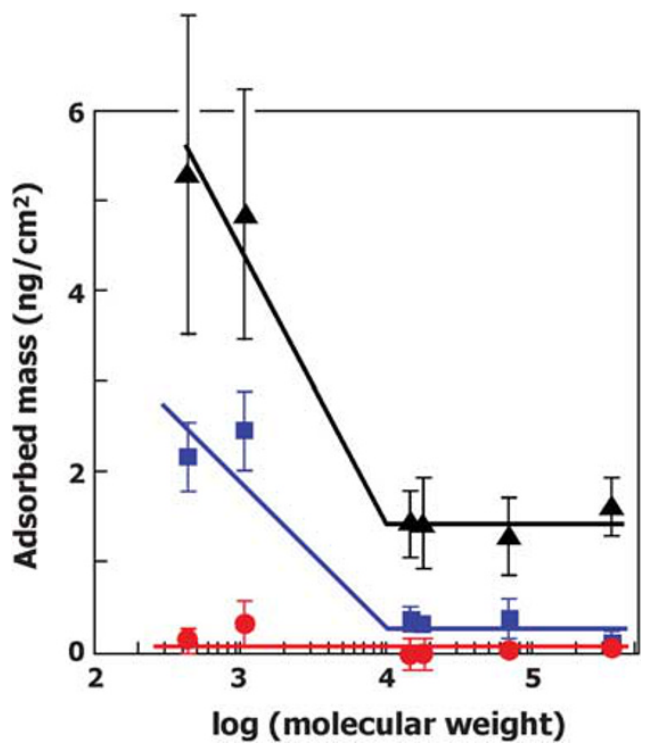

Figure 3 Molecular weight dependency of the nonspecific adsorption of proteins and peptides on the poly(ethylene glycol) (PEG) chain surface and the values given by the carboxyl-dextran sensor chip. The $0.1-\mathrm{mg} \mathrm{ml}^{-1}$ solutions of the proteins and several peptides possessing different molecular weights were analyzed using a conventional PEG (5k) chain surface (squares), a mixed-PEG (5k/2k) chain surface (circles), and a commercial CM5 chip (triangles) blocked by ethanol amine via the active-ester method, $n=4$. Nonspecifically adsorbed mass on PEG surfaces and on CM surfaces was calculated by using $1 \mathrm{RU}$ (unit in BiaCore Instrument) as $0.088 \mathrm{ng} \mathrm{cm}^{-2}$ and as $0.18 \mathrm{ng} \mathrm{cm}^{-2}$. This figure is reproduced from the study by Katsumi et al., 45 by courtesy of the publishers, Elsevier, Amsterdam, The Netherlands.

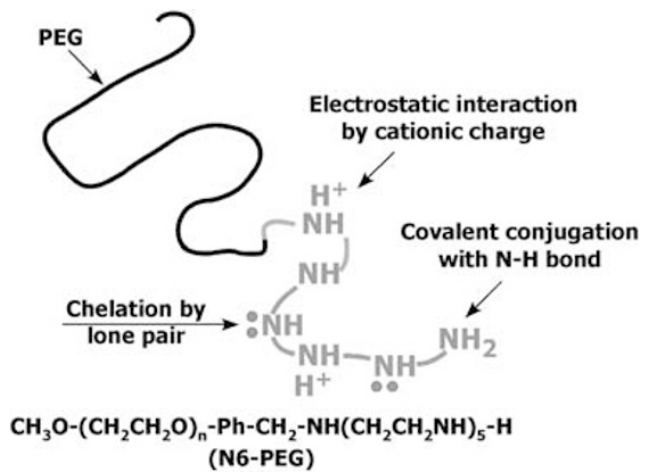

Scheme 2 Pentaethylenehexamine-ended poly(ethylene glycol) (PEG) (N6$P E G$, improves modification efficiencies of active-ester and metal surfaces). 
PEG chains tethered on gold surfaces under physiological conditions, we confirmed that N6-PEG works effectively. Though the coordination of the lone pair of the amino group is fairly low $\left(3-6 \mathrm{kcal} \mathrm{mol}^{-1}\right)^{51,52}$ compared with that of the Au-S linkage (approximately $50 \mathrm{kcal} \mathrm{mol}^{-1}$ ), ${ }^{51}$ polyvalent interactions between the end amino groups of N6-PEG on the gold surface increased their stability significantly. The long-term stability of oligoamine-ended PEG on gold surfaces was confirmed by using gold colloid under physiological conditions. ${ }^{28,39,40,53,54}$

Modified N6-PEG can be applied for other surfaces, especially for active-ester surfaces. ${ }^{16,55,56}$ Because of the covalent conjugation of PEG on active-ester surfaces, mono-amine-ended PEG is commonly used. Though theoretically sound, this technique is not sufficiently suitable for the construction of a densely packed PEG-chain-tethered surface. When N6-PEG is used, the chain density becomes three times higher than that of mono-amine-ended PEG. During the PEG modification process, the number of immobilized PEG chains increases, which tends to prevent access to PEG chains due to the entropically repulsive force. This is why it is difficult to increase the PEG chain density using mono-amine-ended PEG as a modification agent. The pKa values of N6-PEG are in the range of 2-10, which means that N6-PEG possesses both protonated and non-protonated amines in each PEG molecule under the PEG modification conditions. Because active-ester surfaces possess a negative charge, N6-PEG produces an electrostatically attractive force, which is totally different from the mono-amine-ended PEG system. In the case of monoamine-ended PEG, protonated PEG covers the surface, which prevents the nucleophilic attack of non-protonated mono-amine-ended PEG. N6-PEG is now commercially available from JSR, Tokyo, Japan.

\section{CONSTRUCTION OF PROTEIN/PEG HYBRID SURFACES AND THEIR PERFORMANCES}

Enzyme-linked immunosorbent assay is a popular immunoassay method used to quantify immune complexes attached to a solid phase using microtiter plates. Recently, a new enzyme-linked immunosorbent assay system using immunomagnetic beads has been proposed; ${ }^{55,56}$ namely, ligand-conjugated magnetic beads are used as the solid phase instead of the microtiter plate to improve the effectiveness of the antibody conjugation, along with achieving easy handling. Immunomagnetic assay requires high-performance magnetic particles, which must possess sufficient ligand-binding capacity and dispersion stability, because an extremely small number of target molecules must be detected from crude samples containing various proteins and electrolytes. These contaminants always affect the efficiency and reliability of the assay technique. In addition, nonspecific binding to the particles is often responsible for high background levels and poor sensitivity and selectivity. To solve these problems, effective surface modification is needed.

As stated above, mixed PEG chains tethered to a substrate surface show extremely high non-biofouling character. These mixed PEG tethered chains can be applied to magnetic bead surfaces; indeed, densely packed mixed PEG tethered chains have been constructed to surround pre-immobilized antibodies on magnetic surfaces as shown in Scheme 3. This mixed-PEG-chain-tethered surface, which possesses a short PEG filler layer coupled with long PEG tethered chains, has been confirmed to increase its density and result in almost complete protein rejection of not only high-molecular-weight proteins but also small peptides.

To increase the PEG tethered-chain density on the magnetic bead surface, a fill-in treatment was performed similar to that of the gold sensor chip mentioned above; namely, after a PEG tethered-chain

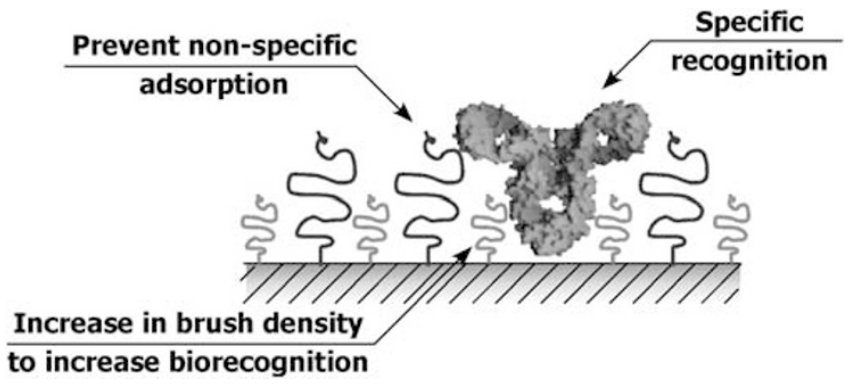

Scheme 3 Antibody/mix poly(ethylene glycol) (PEG) hybridized surface (densely packed mix PEG chain improves not only non-fouling character but also orientation of antibody).

surface was constructed using long PEG chains $(5 \mathrm{kDa})$, short PEG $(2 \mathrm{kDa})$ was immobilized to fill the space between the long preconstructed PEG-chain-tethered surface. The mixed PEG treatments were applied to verify the effectiveness of blocking on the immunomagnetic bead surfaces. As shown in Figure 4a, blocking was effective when the particles were modified with a mixed PEG treatment (PEG $(6 \mathrm{kDa})$ :PEG $(2.5 \mathrm{kDa})=9: 1)$, namely, the surface showed much lower nonspecific binding (4\%) than that coated with N6-PEG (PEG $6 \mathrm{kDa}$ ). This result suggests that the PEG tethering surface, especially that containing mixed PEG, functions as an effective blocking agent. This was attributed to the highly dense PEG chain between the surface antibodies, which repels the nonspecific binding of a secondary antibody. The specific antigen ( $\alpha$-fetoprotein) sensitivity of the magnetic-bead-enzyme-linked immunosorbent assay system was estimated using immunomagnetic beads modified with the mixed N6PEG. It was again confirmed that the sensitivity of the $\alpha$-fetoprotein antibody changes due to the blocking agent. In the case of mixed PEG treatment, this was not significant, but definitely increased the sensitivity compared with the N6-PEG treatment (Figure 4b). Because both low nonspecific binding and high specific sensitivity were achieved for the mixed N6-PEG blocking, the S/N value was remarkably higher than that of BSA, which is used as a conventional blocking agent. Actually, the $4 \%$ mixed PEG blocking showed an S/N value 20 times higher than that of the magnetic beads with BSA blocking (PEG $(6 \mathrm{kDa})$ :PEG $(2.5 \mathrm{kDa})=9: 1$, Figure $4 \mathrm{c})$. These results reveal that a considerable improvement in sensitivity was achieved by using the particles that were treated with mixed N6-PEG.

N6-PEG-coated magnetic beads also showed improved dispersion stability compared with those coated with BSA. After incubation with the cell lysate, the N6-PEG-coated beads were dispersed stably, whereas those coated with BSA and other conventional blocking agents (casein, gelatin; data not shown) resulted in immediate sedimentation within a minute under the same conditions.

Immunoassays are often influenced by nonspecific binding to the solid phase, which is one of the fatal factors that trigger high background and sometimes false positives. Carboxyl-activated immunolattices are usually blocked with amines, such as ethanolamine, aminocaproic acid, glycine or deactivated proteins. However, it is not satisfactory to block the entire charge on a surface, and in some cases, blocking agents accelerate nonspecific protein adsorption. ${ }^{57}$ A certain amount of active esters can be hydrolyzed during the modification process, which reduces blocking efficiency. In the case of a conventional blocking agent such as BSA, it is not sufficient to suppress the nonspecific interaction of proteins on the surface. Because the isoelectric point of BSA is 4.7 , the number of BSA molecules that have access to the particle surface is relatively lower 

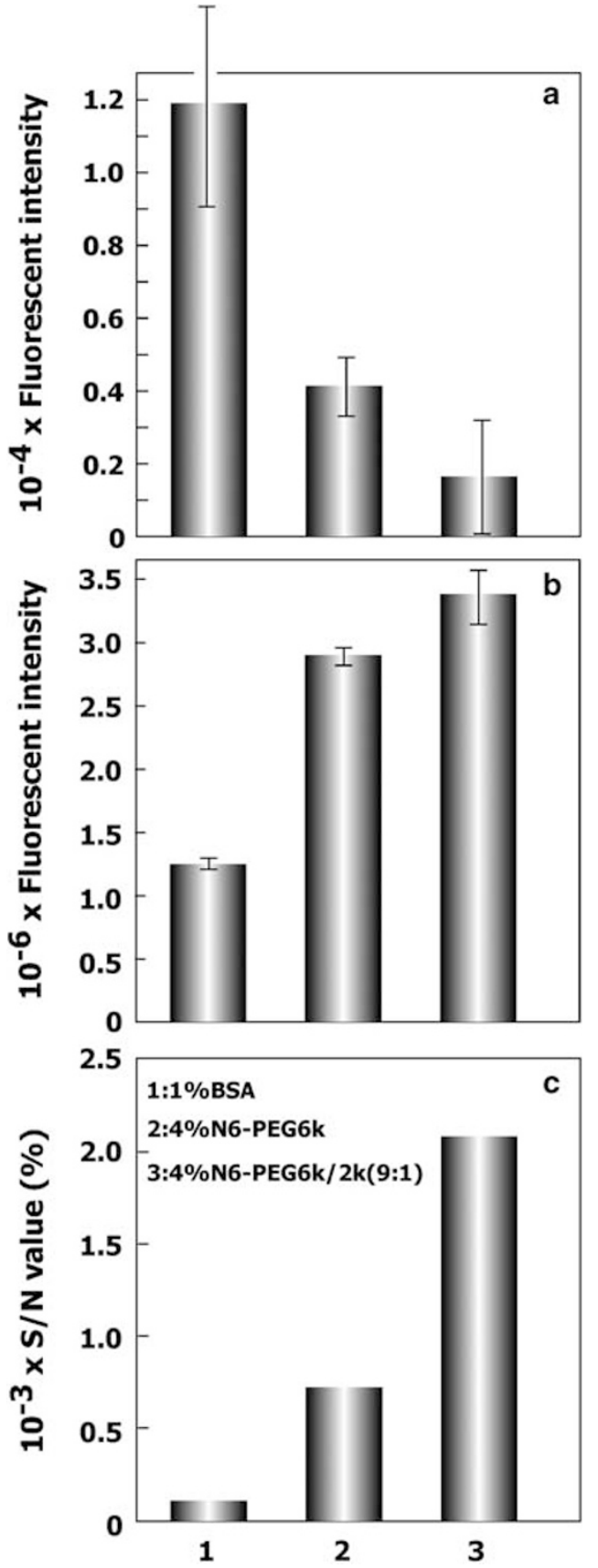

Figure 4 Effect of fill-in treatment of short pentaethylenehexamine at one end (N6-PEG) on (a) nonspecific binding of secondary antibody, (b) immunoassay signal of $\alpha$-fetoprotein sensing, and (c) $\mathrm{S} / \mathrm{N}$ data. 1: Bovine serum albumin (BSA) blocking (1\%); 2: N6-PEG (6 kDa, 4\%) blocking; 3: mixed N6-PEG (PEG (6 kDa):PEG $(2.5 \mathrm{kDa})=9: 1,4 \%)$ blocking; $n=4$. This figure is reproduced from The study by Yukio et al., ${ }^{55}$ by courtesy of the publishers, Elsevier, Amsterdam, The Netherlands.

under the assay conditions (in phosphate-buffered saline, $\mathrm{pH}$ 7.4) due to the electrostatic repulsive force between the two negatively charged surfaces. Thus, the blocking effect of the adsorbed BSA might be substantially reduced.

We have confirmed that the PEG chain density increases with the increasing concentration of the PEG modification agent. ${ }^{14}$ The reactivity of the surface antibody thus increases according to the increase in the PEG tethered-chain density. This tendency was also observed for the mixed PEG treatment; namely, the tethered-chain density increased with the fill-in treatment of a short PEG chain, which increased the surface antibody activity (Figure $4 \mathrm{~b}$ ). The improvement in the surface antibody sensitivity due to the increase in the PEG chain density may be attributed to the enhancement in the performance of the surface antibody by certain factors, such as the orientation of the antibody and the accessibility of antigens. In addition, because the MW of the PEG segment used here was only $6 \mathrm{kDa}$, the accessibility of the corresponding antigen might not be affected, whereas BSA $(68 \mathrm{kDa})$, casein $(>20 \mathrm{kDa})$ and gelatin $(>100 \mathrm{kDa})$ may cover the molecular recognition site on the antibody. Furthermore, it is well known that fraction V of BSA contains dimers or trimers; thus, the molecular recognition sites are readily hindered from accessing the corresponding antigen. These facts may be the reasons for the insufficient blocking capacity and lower sensitivity when immunomagnetic particles are treated with conventionally used blocking agents.

\section{CONSTRUCTION OF OLIGODNA/PEG HYBRID SURFACES AND THEIR PERFORMANCES}

The sensitive and selective detection of target molecules on DNAmodified gold surfaces is one of the powerful analytical methodologies in modern molecular biology. ${ }^{58-62}$ Efficient immobilization protocols yielding high surface coverage, well-established alignment and proper orientation of single-strand DNA (ssDNA) on surfaces as a probe molecule are primary issues in the construction of high-performance DNA-based sensors. The immobilization of probe ssDNA molecules onto a gold surface is generally accomplished by using sulfanylanchored DNA (DNA-SH; It was well known that 'mercapto-' and '-thiol' have been used in the scientific literature as prefix and suffix, respectively. However, according to the 'A Guide to IUPAC Nomenclature of Organic Compounds (Recommendations 1993; Blackwell Scientific publications (1993)', the prefix 'sulfanyl-' is preferred to 'mercapto-' that was used in previous editions of the IUPAC Nomenclature of Organic Chemistry. Therefore, we used 'sulfanyl' as prefix in our manuscripts instead of 'mercapto-' in this paper. You can check this recommendation in the web site http://www.acdlabs.com/iupac/ nomenclature/93/r93_296.htm and http://www.acdlabs.com/iupac/ nomenclature/93/r93_302.htm), which can form a strong bond between a sulfanyl group and a gold surface. ${ }^{63-65}$ However, as shown in recent studies, amine groups on non-hybridized nucleobases in ssDNA can also interact with gold surfaces effectively, becoming unavailable to hybridize with the target DNA molecules on the surface. Thus, extensive efforts to develop surface passivation techniques for the improvement of DNA density and orientation on a gold surface have been made. In a common method for the passivation of gold surfaces, ssDNA-SH-modified gold surfaces can be exposed to a solution containing a small-molecular-weight sulfanyl compound, such as mercapto hexanol or undecanol, which displaces most of the ssDNA-SH from the surface and forces the remaining ssDNA into an upright conformation. ${ }^{66-71}$ Because this method is based on the substitution reaction between ssDNA-SH and sulfanyl-anchored small molecules, the decrease in the surface density of the ssDNA-SH is inevitable, resulting in low sensitivity for the constructed DNA-based sensor.

We have already confirmed the usefulness of the oligoamine anchor ${ }^{15,16,26,27,56,72-76}$ instead of the sulfanyl group anchor ${ }^{7-79}$ for molecular immobilization on gold surfaces and developed polyamineended PEG-modified gold surfaces using PEG-block-poly[2-(N,Ndimethylamino)ethyl methacrylate] (PEG- $b$-PAMA) as an effective 
platform for ssDNA immobilization and detection; ${ }^{73}$ in our scheme, PEG- $b$-PAMA was immobilized onto the gold surface followed by treatment with a ssDNA-SH, resulting in the formation of ssDNA-SH/ PEG- $b$-PAMA co-immobilized gold surface.

Prior to the preparation of an ssDNA/PEG co-immobilized surface, a PEG chain surface was constructed using PEG- $b$-PAMA. To confirm the effectiveness of oligoamine at the end of the PEG chain (so-called block copolymer), we prepared different types of copolymer containing both PEG and PAMA chains, PEG- $b$-PAMA and PAMA-graft-PEG (PAMA-g-PEG; Scheme 4), whose elemental composition ratios were almost the same; comparative studies of PEG- $b$-PAMA and PAMA- $g$ PEG layers on gold surfaces by SPR and X-ray photoelectron spectroscopy (XPS) analyses were conducted. ${ }^{80}$

To evaluate the characteristics of a PEG-polyamine layer constructed on a gold surface, XPS analysis was conducted for PEG- $b$ PAMA- and PAMA-g-PEG-modified gold surfaces. The elemental compositions of the PEG polyamines given as atomic percentages (\%) were close to the expected theoretical compositions predicted by molecular stoichiometry. Additionally, the elemental compositions of $\mathrm{C}, \mathrm{O}$ and $\mathrm{N}$ on the PEG- $b$-PAMA-modified gold surface were very close to those on the PEG-g-PAMA-modified surface, indicating that there was almost no difference in the elemental composition ratios between the synthesized PEG- $b$-PAMA and PAMA- $g$-PEG, as expected from the polymer characterization data.

The data shown in Figure 5 obtained from the standard XPS analysis at a $90^{\circ}$ takeoff angle do not reveal the differences between PEG polyamine layers on gold surfaces. However, angle resolved X-ray photoelectron spectroscopy (ARXPS) analysis, XPS analysis data obtained at several takeoff angles, clearly elucidate the conformational difference between PEG- $b$-PAMA and PAMA- $g$-PEG layers on gold surfaces. Figures $5 \mathrm{a}$ and $\mathrm{b}$ show the ARXPS data of PEG- $g$-PAMA- and PAMA- $b$-PEG-modified gold surfaces, respectively, where the longitudinal axis is the ratio between nitrogen and carbon atomic composition (N/C), and the horizontal axis represents the takeoff angles of photoemission. In the case of the PEG-g-PAMA-modified gold surface, the N/C values at all of the takeoff angles (from $0^{\circ}$ to $90^{\circ}$ ) were almost the same, indicating that PAMA segments and PEG segments were distributed over all regions of the constructed polymer layer, as illustrated in the inset of Figure $5 \mathrm{a}$. However, in the case of the PEG- $b$-PAMA-modified gold surface, a decrease in the N/C value with decreasing takeoff angle was observed. At takeoff angles ranging from $45^{\circ}$ to $90^{\circ}$, an N/C value of 0.95 was obtained, which was almost the

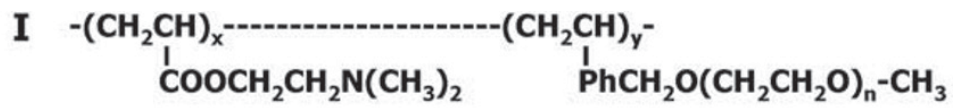

Poly[2-(N,N-dimethylamino)ethyl methacrylate]-g-poly(ethylene glycol)

\author{
II $\mathrm{CH}_{3} \mathrm{O}-\left(\mathrm{CH}_{2} \mathrm{CH}_{2} \mathrm{O}\right)_{n}-\left(\mathrm{CH}_{2} \mathrm{CH}\right)_{m}-\mathrm{H}$ \\ $\mathrm{COOCH}_{2} \mathrm{CH}_{2} \mathrm{~N}\left(\mathrm{CH}_{3}\right)_{2}$ \\ Poly(ethylene glycol)-b-poly[2-(N,N-dimethylamino)ethyl methacrylate]
}
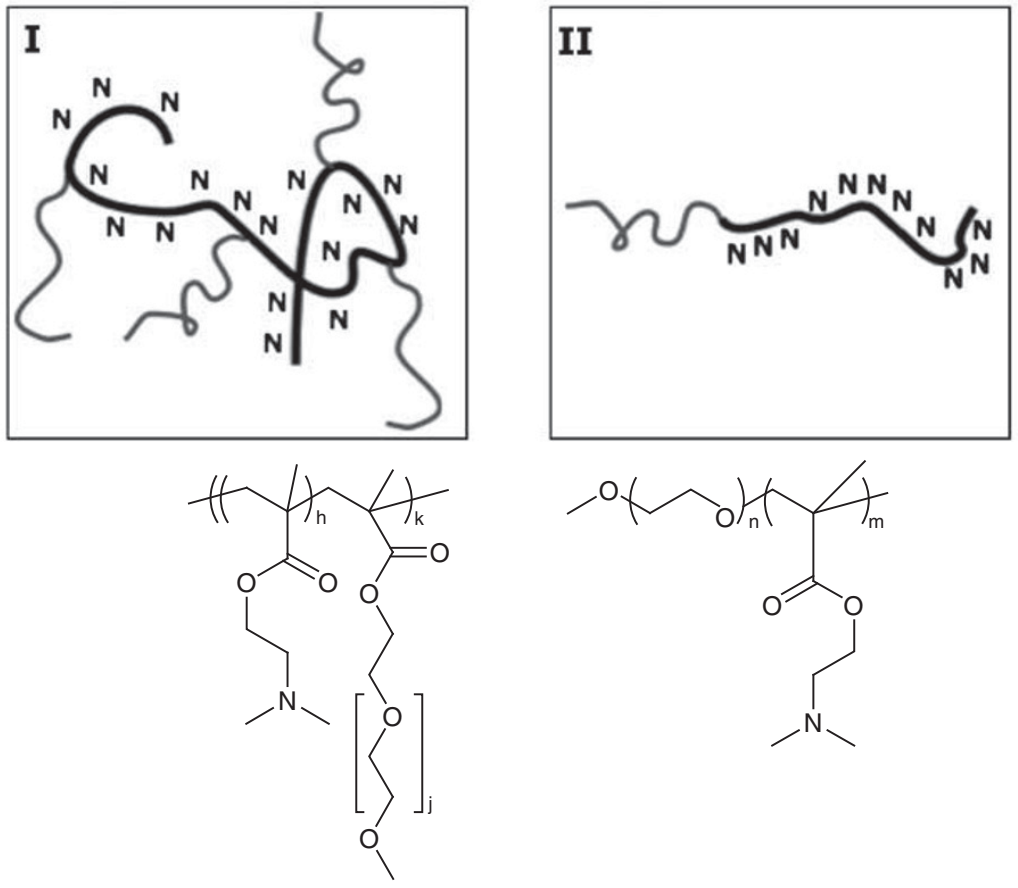

Scheme 4 Polymers used in this study (right: poly(ethylene glycol) (PEG)-block-poly[2- $N, N$-(dimethylamino)ethyl methacrylate], PEG- $b$-PAMA, and left: poly[2-N,N-(dimethylamino)ethyl methacrylate]-graft-PEG, PEG-g-PAMA). 


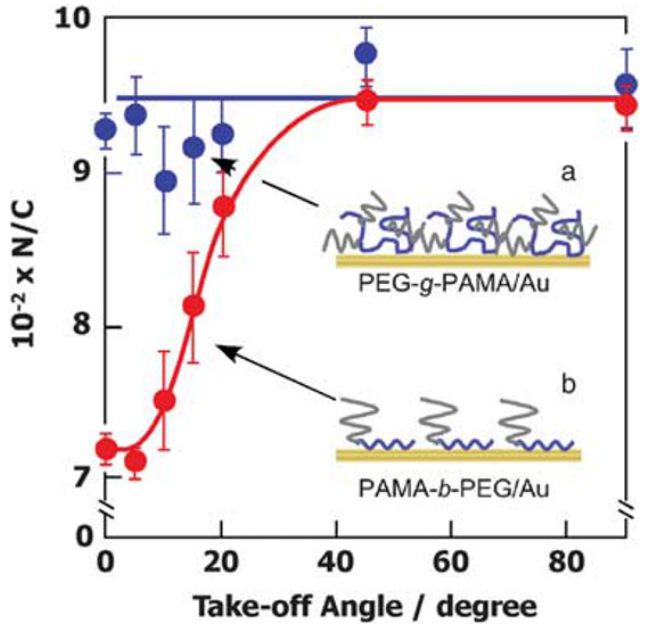

Figure 5 ARXPS data of the (a) poly(ethylene glycol) (PEG)-graft-poly[2( $N, N$-dimethylamino)ethyl methacrylate] (PEG-g-PAMA)- and (b) PAMA- $b$ PEG-modified gold surfaces. The illustrations inserted in these figures show the possible structures of the PEG polyamine layer constructed on the gold surface, where the PAMA segments and the PEG segments in PEG-g-PAMA were distributed over all regions of the constructed polymer layer, while the PAMA segments in PEG- $b$-PAMA were concentrated at the gold surface. This figure is reproduced from Keitaro et al., 80 by courtesy of the publishers, American Chemical Society, USA.

same value as that for the PEG-g-PAMA-modified gold surface at takeoff angles from $0^{\circ}$ to $90^{\circ}$ (Figure 5a). Interestingly, the N/C values decreased dramatically with decreasing takeoff angle from $45^{\circ}$ to $0^{\circ}$ and finally reached 0.72 . This result indicates a decrease in the nitrogen content in the upper level of the constructed polymer layer, namely, the inhomogeneous distribution of PAMA and PEG segments in the constructed PEG- $b$-PAMA polymer layer on the gold surface. Therefore, in the case of the PEG- $b$-PAMA-modified gold surface, the PAMA segment of PEG- $b$-PAMA was concentrated on the gold surface, and the PEG brush layer may have been constructed on the surface as described in the inset in Figure 5b. The adsorption properties of PEG-chain-containing graft copolymers onto inorganic substrates have been studied by the groups of Textor, ${ }^{81}$ Spencer $^{82}$, and their co-workers, where the adsorption stability of these copolymers was observed to be strongly affected by the $\mathrm{pH}$, salt concentration and their grafting density. In this study and our companion article, ${ }^{80}$ we reported for the first time the direct observations of homogeneous/ inhomogeneous distributions of PEG and polyamine segments in the polymer layers on flat gold surfaces, where polyamine segments functioned as an anchor for molecular immobilization on the surface and at least six units of amine groups were needed for the strong chemisorption of polyamine on a gold substrate. ${ }^{26}$

Using PEG- $b$-PMA block copolymers with different chain lengths, we then prepared an ssDNA/PEG- $b$-polyamine hybrid surface on a gold surface. It is interesting to note that the PEG- $b$-PAMA modification increased the amount of immobilized ssDNA-SH on the gold surface to 2-5 times as much as that on a bare gold surface, where the effective and selective detection of the complementary ssDNA was accomplished by the ssDNA-SH/PEG- $b$-PAMA (5k/5k) co-immobilized surface.

Figure 6a shows the angle shift SPR data for ssDNA-SH immobilization on a PEG-chain-tethered surface pre-modified by PEG-SH, N6-PEG or PEG- $b$-PAMA. When the probe ssDNA-SH contacted the N6-PEG(5 kDa)-pre-immobilized surface, almost no shifts in the SPR
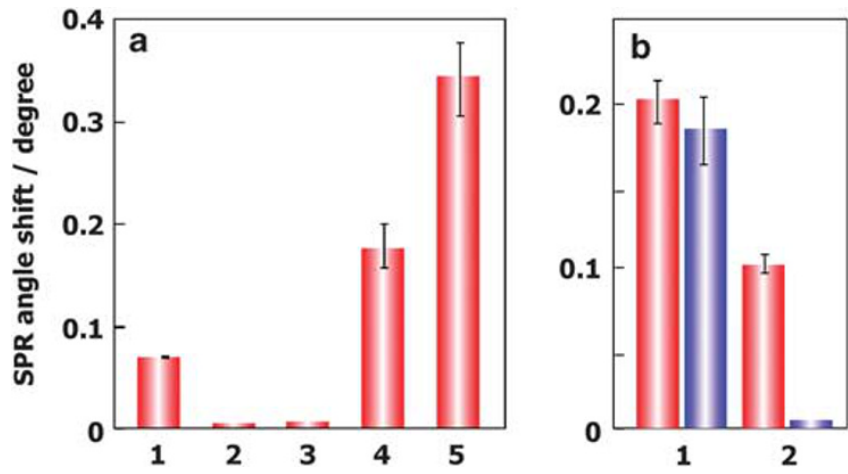

Figure 6 (a) Surface plasmon resonance (SPR) angle shift of single-stranded DNA (ssDNA)-sulfanyl (SH) immobilization on a bare and poly(ethylene glycol) (PEG)-modified gold surface: (1) bare, (2) PEG-SH, (3) pentaethylenehexamine-ended PEG (N6-PEG), (4) PEG- $b$-PAMA (PEG-blockpoly[2-( $N, N$-dimethylamino)ethyl methacrylate], $5 \mathrm{k} / 5 \mathrm{k}$ ) and (5) PEG- $b$ PAMA ( $5 \mathrm{k} / 10 \mathrm{k}$ ). (b) SPR angle shift changes of ssDNA adsorption (red bar), followed by washing with $1 \mathrm{~m} \mathrm{NaCl}$ solution (blue bar) on the PEG-b-PAMA (5k/5k)-modified gold surface: (1) ssDNA-SH and (2) ssDNA (without the $\mathrm{SH}$ group). This figure is reproduced from Yoshimoto et al., ${ }^{73}$ by courtesy of the publishers, Chemical Society of Japan.

angle were observed, as was the case with the PEG-SH-modified surface. On the contrary, the shifts in the SPR angle after ssDNA-SH adsorption on the PEG- $b$-PAMA (5k/5k) and PEG- $b$-PAMA (5k/10k)modified surfaces were two- and fivefold higher than those on the bare gold surface, respectively. Taking into account the shifts in the SPR angle after polymer adsorption, these facts indicate that the amount of ssDNA-SH adsorbed onto a gold surface can be increased by modifying the oligoamine chain length of the PEG-oligoamine block copolymer.

To confirm ssDNA-SH immobilization via the Au-S linkage on the PEG-oligoamine-modified surface, the immobilization behavior of ssDNA ( $5^{\prime}-(\mathrm{T})_{20}$-GCCACCAGC-3'), which has no mercapto group at the $5^{\prime}$ end, on the PEG- $b$-PAMA (5k/5k)-modified surface was examined. As shown in Figure 6b, SPR angle shifts of $0.2^{\circ}$ and $0.1^{\circ}$ were observed when ssDNA-SH and ssDNA were adsorbed onto the PEG- $b$-PAMA (5k/5k)-modified surface, respectively. Almost no angle shift change was observed when the ssDNA-SH/PEG co-modified surface was washed with $1 \mathrm{M} \mathrm{NaCl}$ solution. In contrast, the $0.1^{\circ} \mathrm{SPR}$ angle shift disappeared almost completely on the ssDNA/PEG comodified surface as a result of the $1 \mathrm{M} \mathrm{NaCl}$ wash. These results indicate that the electrostatically adsorbed ssDNA on the PEG- $b$ PAMA (5k/5k)-modified surface was easily removed by the $1 \mathrm{M} \mathrm{NaCl}$ solution, while the ssDNA-SH was strongly immobilized on the PEG$b$-PAMA-modified gold surface via the covalent Au-S linkage. These adsorption behaviors of ssDNA and ssDNA-SH indicate that the constructed DNA-SH- $b$-PEG-oligoamine co-immobilized surfaces are stable under physiological conditions and support the immobilization model shown in Scheme 5.

To assess the hybridyzation behavior of the probe ssDNA immobilized on the PEG- $b$-oligoamine-modified surface, the SPR signal of the target ssDNA (5'-GCTGGTGGC-3'; complementary ssDNA) and single-base-substituted ssDNA (5'-GCTGTTGGC-3'; mismatched ssDNA) was analyzed on the bare and the PEG- $b$-PAMA $(5 \mathrm{k} / 5 \mathrm{k})$ modified gold surface (Figure 7). Although the same amount of ssDNA-SH was immobilized on both surfaces (in this experiment, the immobilized amounts of ssDNA-SH on both surfaces were adjusted to the $0.2^{\circ}$ SPR angle shift), the angle shift of complementary ssDNA adsorption on the PEG- $b$-PAMA-modified surface was much 


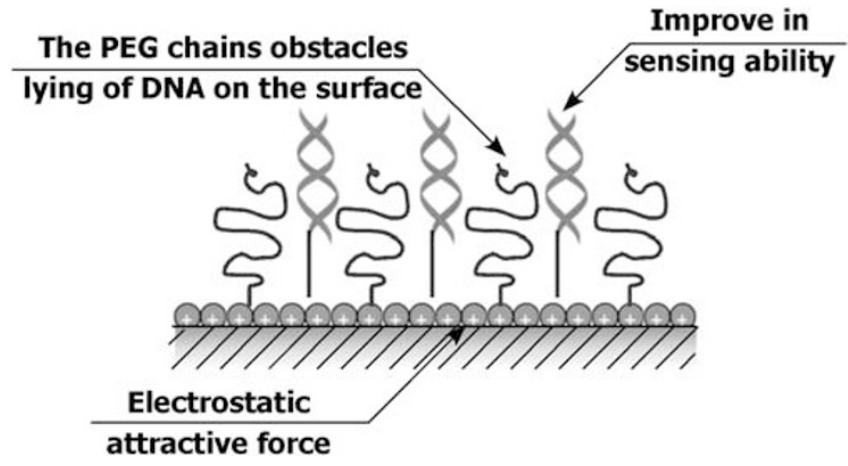

Scheme 5 OligoDNA/poly(ethylene glycol) (PEG) hybridized surface (densely packed mix PEG chain improves not only non-fouling character but also orientation of oligoDNA).

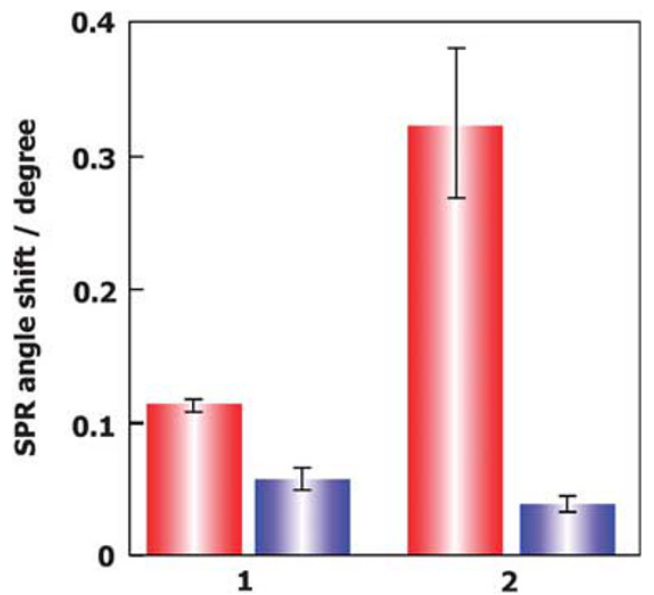

Figure 7 Surface plasmon resonance (SPR) sensing of complementary single-stranded DNA (ssDNA, red bar) and mismatched ssDNA (blue bar) on a probe ssDNA-sulfanyl (SH)-immobilized gold surface: (1) bare and (2) a poly(ethylene glycol) (PEG)/poly[2-( $N, N$-dimethylamino)ethyl methacrylate] (PAMA, 5k/5k)-modified surface. This figure is reproduced from Yoshimoto et al., ${ }^{73}$ by courtesy of the publishers, Chemical Society of Japan.

higher than that on the bare gold surface, presumably due to the difference in the probe ssDNA orientations on the two surfaces. Thus, PEG- $b$-PAMA block copolymer immobilized on a gold surface may inhibit the interaction between the gold interface and the nucleobases in ssDNA, ${ }^{6}$ resulting in the standing conformation of the immobilized ssDNA, which recovers its ability to hybridize with the complementary ssDNA. In contrast, the angle shift in the adsorption of the mismatched ssDNA on the PEG/ssDNA surface was lower than that on the bare gold surface. The PEG tethered chains on the PEG/ssDNA mixed surface are considered to reduce the nonspecific adsorption of the mismatched ssDNA. Consequently, the $\mathrm{S} / \mathrm{N}$ value of the constructed PEG/ssDNA mixed-chain-tethered surface was more than four times higher than that of the bare gold surface.

\section{MISCELLANEOUS PEG-CHAIN-TETHERED SURFACES}

Using mixed PEG tethered chains on substrate surfaces, several other unique surfaces have been constructed. When mixed PEG chains were constructed to surround an enzyme pre-immobilized on a gold nanoparticle surface, the thermal stability of the surface increased significantly, ${ }^{83,84}$ thus enabling the development of thermally stable

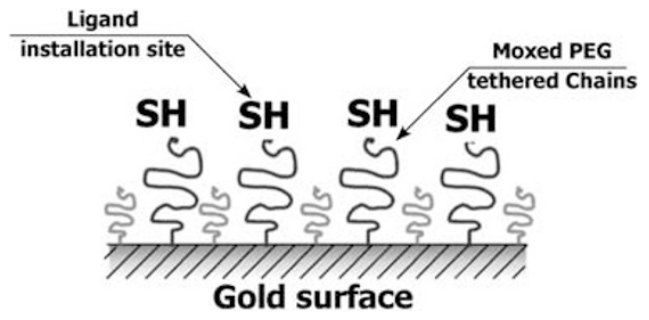

Scheme 6 Mixed-poly(ethylene glycol) (PEG) tethered-chain possessing freesulfanyl (SH) group at the PEG chain end on gold surface (PEG chain improves non-fouling character and prevents loop formation of the $\mathrm{SH}$-end group on gold surface).

enzyme complexes. A sulfanyl-terminated PEG brush layer was constructed on a gold sensor platform by consecutive treatment with a sulfanyl-ended semitelechelic PEG (MeO-PEG-SH, $2 \mathrm{kDa}$ ) and a sulfanyl-ended telechelic PEG (5 kDa, SH-PEG-SH). ${ }^{85}$ It is based on a mixed-PEG-brush formation from the longer SH-PEG-SH (5k) and the shorter MeO-PEG-SH (2k), where the pre-immobilized shorter MeO-PEG-SH (2k) prevents loop formation in the longer SH-PEG$\mathrm{SH}(5 \mathrm{k})$ on the surface and the free sulfanyl group at one end of the longer SH-PEG-SH is exposed to the mixed-PEG-chain-tethered surface. From the experimental results obtained from SPR analysis, it became apparent that the immobilization density and the orientation of the longer SH-PEG-SH (5k) on the gold surface could be controlled by the amount of pre-immobilized shorter MeO-PEG-SH (2 k; Scheme 6). A two-dimensional microarray of $10000(100 \times 100)$ hepatocyte heterospheroids, underlain with endothelial cells, was successfully constructed with $100-\mu \mathrm{m}$ spacing in an active area of $20 \times 20 \mathrm{~mm}$ on microfabricated glass substrates that were coated with mixed PEG brushes. Co-cultivation of hepatocytes with endothelial cells was essential to stabilize hepatocyte viability and liver-specific functions, allowing us to obtain hepatocyte spheroids with a diameter of $100 \mu \mathrm{m}$ that functioned as a miniaturized liver to secrete albumin for at least 1 month. The mixed-PEG-chain-tethered chain layer has an important role in preventing the overgrowth of the underlayer of endothelial cells from the $100-\mu \mathrm{m}$ domain to the surrounding surface area.

\section{ACKNOWLEDGEMENTS}

This research was partially supported by a Grant-in-Aid for Scientific Research on Innovative Areas (Soft Interface), no. 20106011, from the Ministry of Education, Science, Sports and Culture of Japan. Most of this study was conducted by our colleagues and students. We sincerely appreciate their devoted cooperation.

1 Schultz, J., Mrksich, M., Bhatia, S. N. \& Brady, D. J. Biosensing: International Research and Development (Springer, Tokyo, 2006).

2 Edwards, R. Immunodiagnostics. Practical Approach (Practical Approach Series) (Oxford University Press, London, 1999).

3 Wild, D. G. (ed.). The Immunoassay Handbook 3rd edn (Elsevier, Amsterdam, 2005).

4 Kurien, B. T. \& Hal Scofield, R. (ed.). Protein Blotting and Detection: Methods and Protocols (Methods in Molecular Biology) (Humana Press, New York, 2009).

5 Fung, E. Protein Arrays Methods and Protocols (Methods in Molecular Biology) (Humana Press, New York, 2010).

6 Hermanson, G. T. Bioconjugate Techniques 2nd edn (Academic Press, Waltham, 2008).

7 Becker, G. S., Copeland, C. W. \& Lister, S. A. Mad Cow Disease Bovine Spongiform Encephalopathy (Nova Publishers, Hauppauge, 2008).

8 Mori, Y., Nagaoka, S., Takiuchi, H., Kikuchi, T., Noguchi, N., Tanzawa, H. \& Noishiki, Y. A new antithrombogenic materials with long polyethylene oxide chain. Trans. Am. Soc. Artif. Intern. Organs 28, 459 (1982) Scheme 5: OligoDNA/poly(ethylene glycol) (PEG) hybridized surface (densely packed mix PEG chain improves not only non-fouling character but also orientation of oligoDNA). Figure 7: Surface plasmon resonance (SPR) 
sensing of complementary single-stranded DNA (ssDNA, red bar) and mismatched ssDNA (blue bar) on a probe ssDNA-sulfanyl ( $\mathrm{SH}$ )-immobilized gold surface: (1) bare and (2) a poly(ethylene glycol) (PEG)/poly[2-(N,N-dimethylamino)ethyl methacrylate] (PAMA, 5k/5k)-modified surface. This figure is reproduced from Yoshimoto et al., 73 by courtesy of the publishers, Chemical Society of Japan. Scheme 6: Mixed-poly(ethylene glycol) (PEG) tethered-chain possessing freesulfanyl (SH) group at the PEG chain end on gold surface (PEG chain improves non-fouling character and prevents loop formation of the SH-end group on gold surface).

9 Jeon, S. I. \& Andrade, J. D. Protein-surface interactions in the presence of polyethylene oxide: II. Effect of protein size. J. Colloid Interface Sci. 142, 159-166 (1991).

10 Gölander, C.- G., Herron, J. N., Lim, K., Claesson, P., Stenius, P. \& Andrade, J. D. in Poly(Ethyleneglycol) Chemistry, Biotechnical and Biomedical Applications (ed. Harris, J. M.) 221-245 (Plenum, New York, 1992).

11 Lina, Y. S., Hlady, V. \& Gölander1, C.- G. Colloids and surfaces B. Biointerfaces 3, 49-62 (1994).

12 Pasche, S., Volrols, J., Griesser, H. J., Spencer, N. D. \& Textor, M. Effects of ionic strength and surface charge on protein adsorption at PEGylated surfaces. J. Phys. Chem. B 109, 17545-17552 (2005).

13 Otsuka, H., Nagasaki, Y. \& Kataoka, K. Surface characterization of functionalized polylactide through the coating with heterobifunctional poly(ethylene glycol)/polylactide block copolymers. Biomacromolecules 1, 39-48 (2000).

14 Uchida, K., Otsuka, H., Kaneko, M., Kataoka, K. \& Nagasaki, Y. A reactive poly(ethylene glycol) layer to achieve specific surface plasmon resonance sensing with a high S/N ratio: the substantial role of a short underbrushed peg layer in minimizing nonspecific adsorption. Anal. Chem. 77, 1075-1080 (2005).

15 Nagasaki, Y. Engineering of poly(ethylene glycol) chain-tethered surfaces to obtain high performance bionanoparticles. Sci. Technol. Adv. Mater. 11, 054505 (2010).

16 Yuan, X., Fabregat, D., Yoshimoto, K. \& Nagasaki, Y. Design of highly functional antiferritin-immunolatex by hybridization of antiferritin/mixed-PEG polymers onto polystyrene submicroparticles. Biomaterials (Symp. Ser.) 1054, 243-258 (2010) (Chapter 13).

17 Harbers, G. M., Barber, T. A., Healy, K. E., Stile, R. A. \& Sumner, D. R. in Biomimetic Materials and Design (eds Dillow, A. \& Lowman, A.) 55-90 (Marcel Dekker, New York, 2002).

18 Goddarda, J. M. \& Hotchkiss, J. H. Polymer surface modification for the attachment of bioactive compounds. Prog. Poly. Sci. 32, 698-725 (2007).

19 Yoshimoto, K., Ichino, M. \& Nagasaki, Y. Inverted pattern formation of cell microarrays on poly(ethylene glycol) (PEG) gel patterned surface and construction of hepatocyte spheroids on unmodified PEG gel microdomains. Lab on a Chip 9, 1286-1289 (2009).

20 Kojima, R., Yoshimoto, K., Miyoshi, H. \& Nagasaki, Y. Spheroid array of fetal mouse liver cells constructed on a PEG-gel micropatterned surface: upregulation of hepatic functions by co-culture with nonparenchymal liver cells. Lab on a Chip 9, 1991-1993 (2009).

21 Leckband, D., Sheth, S. \& Halperin, A. Grafted poly(ethylene oxide) brushes as nonfouling surface coatings. J. Biomater. Sci. Poly. Ed. 10, 1125-1147 (1999).

22 Paschalis Alexandridis, T. A. H. Poly(ethylene oxide)-poly(propylene oxide)-poly(ethylene oxide) block copolymer surfactants in aqueous solutions and at interfaces: thermodynamics, structure, dynamics, and modeling. Colloids Surf. A: Physicochem. Eng. Aspects 96, 1-46 (1995).

23 Otsuka, H., Nagasaki, Y. \& Kataoka, K. Characterization of aldehyde-PEG tethered surfaces: influence of PEG chain length on the specific biorecognition. Langmuir 20, 11285-11287 (2004)

24 Youngblood, J. P., Andruzzi, A., Ober, C. K., Hexemer, A., Kramer, E. J., Callow, J. A., Finlay, J. A. \& Callow, M. E. Coatings based on side-chain ether-linked poly(ethylene glycol) and fluorocarbon polymers for the control of marine biofouling. Biofouling 19 (Suppl), 91-98 (2003).

25 Elbert, D. L. \& Hubbell, J. A. Self-assembly and steric stabilization at heterogeneous, biological surfaces using adsorbing block copolymers. Chem. Biol. 5, 177-183 (1998).

26 Miyamoto, D., Oishi, M., Kojima, K., Yoshimoto, K. \& Ngasaki, Y. Completely dispersible PEGylated gold nanoparticles under physiological conditions: modification of gold nanoparticles with precisely controlled PEG-b-polyamine. Langmuir 24, 5010-5017 (2008)

27 Yoshimoto, K., Nishio, M., Sugasawa, H. \& Nagasaki, Y. Direct observation of adsorption induced inactivation of antibody fragments surrounded by mixed-peg layer on a gold surface. J. Am. Chem. Soc. 132, 7982-7989 (2010).

28 Ning-Ping Huang, J. V., De Paul, S. M., Textor, M. \& Spencer, N. D. Biotin-derivatized poly(L-lysine)-g-poly(ethylene glycol): a novel polymeric interface for bioaffinity sensing. Langmuir 18, 220-230 (2002).

29 Bailey, F. E. J. V. K. Alkylene Oxides and Their Polymers (Marcel Dekker Inc., New York, 1990).

30 Gibson, M. Pharmaceutical Preformulation and Formulation: A Practical Guide from Candidate Drug Selection to Commercial Dosage Form (Drugs and the Pharmaceutical Sciences) (Informa Healthcare, London, 2009).

31 Hustedt, H., Johansson, G. \& Tjerneld, F. Aqueous Two-Phase Separation Systems (Kluwer Academic Publishers, Dordrecht, Netherlands, 1990).

32 E Glass, J. Hydrophilic Polymers: Performance with Environmental Acceptance (American Chemistry Society, Washington, DC, 1996).

33 Deutscher, M. P. Guide to protein purification. Meth Enzymol 182, 646-659 (1990). 34 Sowers, A. E. Cell Fusion (Plenum Press, New York, NY, 1987).

35 Shalaby, S. W., McCormick, C. L. \& Butler, G. B. (ed.). Water-Soluble, Polymers, Synthesis, Solution Properties, and Applications, ACS Symposium Series, Vol. 467 (American Chemical Society, 1991).
36 Sawhney, A. S., Chandrashekhar, P. P. \& Hubbell, J. A. Interfacial photopolymerization of polyethylene glycol-based hydrogels upon alginate-poly(I-lysine) microcapsules for enhanced biocompatibility. Biomaterials 14, 1008-1016 (1993).

37 Efremova, N. V., Hung, Y., Peppas, N. A. \& Leckband, D. E. Direct measurement of interactions between tethered polyethylene glycol chains and adsorbed mucin layers. Langmuir 18, 836-845 (2002).

38 Martin Malmsten, D. M. Interfacial behavior of 'new' poly(ethylene oxide)-containing copolymers. J. Biomater. Sci. Polym. Ed. 10, 1075-1087 (1999).

39 Blaettler, T. M., Marcus Textor, S. P. \& Griesser, H. J. High salt stability and protein resistance of poly(L-lysine)-g-poly(ethylene glycol) copolymers covalently immobilized via aldehyde plasma polymer interlayers on inorganic and polymeric substrates. Langmuir 22, 5760-5769 (2006).

40 Chen, Y., Kang, E. T., Neoh, K. G., Wang, P. \& Tan, P. K. L. Surface modification of polyaniline film by grafting of poly(ethylene glycol) for reduction in protein adsorption and platelet adhesion. Synthetic Met. 110, 47-55 (2000).

41 Tsujii, Y., Ejaz, M., Yamamoto, S., Ohno, K., Urayama, K. \& Fukuda, T. in Structure and Properties of High-Density Polymer Brushes (eds Advincula, R. C., Brittain, W. J., Caster, K. C. \& Rühe, J.) (Polymer Brushes, Wiley-VCH, Weinheim, 2004).

42 Sofia, S. J., Premnath, V. \& Merrill, E. W. Poly(ethylene oxide) grafted to silicon surfaces: grafting density and protein adsorption. Macromolecules 31, 5059-5070 (1998).

43 Raju Francis, A. M. S., Carino, S. R., Logan, J. L., Underhill, R. S., Angot, S., Taton, D., Gnanou, Y. \& Duran, R. S. Aggregation and surface morphology of a poly(ethylene oxide)-block-polystyrene three-arm star polymer at the air/water interface studied by AFM. Macromolecules 35, 6483-6485 (2002)

44 Emoto, K., Michihiro, I., Nagasaki, Y. \& Kataoka, K. Functionality of polymeric micelle hydrogels with organized three-dimensional architecture on surfaces. J. Am. Chem. Soc. 122, 2653-2654 (2000).

45 Uchida, K., Hoshino, Y., Tamura, A., Yoshimoto, K., Kojima, S., Yamashita, K., Yamanaka, I., Otsuka, H., Kataoka, K. \& Nagasaki, Y. Creation of a mixed poly (ethylene glycol) tethered-chain surface for preventing the non-specific adsorption of proteins and peptides. Biointerphases 2, 126 (2007).

46 Otsuka, H., Akiyama, Y., Nagasaki, Y. \& Kataoka, K. Quantitative and reversible lectininduced association of gold nanoparticles modified with $\alpha$-lactosyl- $\omega$-mercaptopoly(ethylene glycol). J. Am. Chem. Soc. 123, 8226-8230 (2001).

47 Wuelfing, W. P., Gross, S. M., Miles, D. T. \& Murray, R. W. Nanometer gold clusters protected by surface-bound monolayers of thiolated poly(ethylene glycol) polymer electrolyte. J. Am. Chem. Soc. 120, 12696-12697 (1998).

48 Bearinger, J. P., Terrettaz, S., MicheL, R., Tirelli, N., Vogel, H., Textor, M. \& Hubbell, J. A. Chemisorbed poly(propylene sulphide)-based copolymers resist biomolecular interactions. Nat. Mater. 2, 259 (2003).

49 Cooper, E. \& Leggett, G. J. Static secondary ion mass spectrometry studies of selfassembled monolayers: influence of adsorbate chain length and terminal functional group on rates of photooxidation of alkanethiols on gold. Langmuir 14, 4795 (1998).

50 Castner, D. G., Hinds, K. \& Grainger, D. W. X-ray photoelectron spectroscopy sulfur 2p study of organic thiol and disulfide binding interactions with gold surfaces. Langmuir 12, 5083 (1996).

51 Pong, B.- K., Lee, J.- Y. \& Trout, B. L. First principles computational study for understanding the interactions between ssDNA and gold nanoparticles: adsorption of methylamine on gold nanoparticulate surfaces. Langmuir 21, 11599 (2005).

52 Felice, R. D. \& Selloni, A. First principles computational study for understanding the interactions between ssDNA and gold nanoparticles: adsorption of methylamine on gold nanoparticulate surfaces. J. Chem. Phys. 120, 4906-4914 (2004).

53 Ishii, T., Otsuka, H., Kataoka, K. \& Nagasaki, Y. Preparation of functionally PEGylated gold nanoparticles with narrow distribution through autoreduction of auric cation by a-biotinyl-PEG-block-[poly(2-(N,N-dimethylamino)ethyl methacrylate)]. Langmuir 20, 561-564 (2004).

54 Furusho, H., Kitano, K., Hamaguchi, S. \& Nagasaki, Y. Preparation of stable waterdispersible pegylated gold nanoparticles assisted by nonequilibrium atmospheric pressure plasma jets. Chem. Mater. 21, 3526-3535 (2009).

55 Nagasaki, Y., Kobayashi, H., Katsuyama, Y., Jomura, T. \& Sakura, T. Enhanced immunoresponse of antibody/mixed-PEG co-immobilized surface construction of high-performance immunomagnetic ELISA system. J. Colloid Interface Sci. 309, 524-530 (2007).

56 Xiaofei, Y., Yoshimoto, K. \& Nagasaki, Y. High-performance immunolatex possessing a mixedpeg/antibody coimmobilized surface: highly sensitive ferritin immunodiagnostics. Anal. Chem. 81, 1549-1556 (2009).

57 Soukka, T., Paukkunen, J., Harma, H., Lonnberg, S., Lindroos, H. \& Lovgren, T. Supersensitive time-resolved immunofluorometric assay of free prostate-specific antigen with nanoparticle label technology. Clin. Chem. 47, 1269 (2001).

58 Langer, R. \& Tirrell, D. A. Designing materials for biology and medicine. Nature $\mathbf{4 2 8}$ 487-492 (2004)

59 Cooper, M. A. \& Singleton, V. T. A survey of the 2001 to 2005 quartz crystal microbalance biosensor literature: applications of acoustic physics to the analysis of biomolecular interactions. J. Mol. Recognit. 20, 154-184 (2007).

60 Belosludtsev, Y., Iverson, B., Lemeshko, S., Eggers, R., Wiese, R., Lee, S., Powdrill, T. \& Hogan, M. DNA microarrays based on noncovalent oligonucleotide attachment and hybridization in two dimensions. Anal. Biochem. 292, 250-256 (2001).

61 Petrovykh, D. Y., Kimura-Suda, H., Tarlov, M. J. \& Whitman, L. J. Quantitative characterization of DNA films by X-ray photoelectron spectroscopy. Langmuir 20, 429-440 (2004).

62 Höök, F., Ray, A., Nordén, B. \& Kasemo, B. Characterization of PNA and DNA immobilization and subsequent hybridization with DNA using acoustic-shear-wave attenuation measurements. Langmuir 17, 8305-8312 (2001). 
63 Love, J. C., Estroff, L. A., Kriebel, J. K., Nuzzo, R. G. \& Whitesides, G. M. Selfassembled monolayers of thiolates on metals as a form of nanotechnology. Chem. Rev. 105, 1103-1169 (2005).

64 Ulman, A. Formation and structure of self-assembled monolayers. Chem. Rev. 96, 1533-1554 (1996).

65 Prime, K. L. \& Whitesides, G. M. Self-assembled organic monolayers: model systems for studying adsorption of proteins at surfaces. Science 252, 1164-1167 (1991).

66 Gong, P., Lee, C.- Y., Gamble, L. J., Castner, D. G. \& Grainger, D. W. Hybridization behavior of mixed DNA/alkylthiol monolayers on gold: characterization by surface plasmon resonance and 32p radiometric assay. Anal. Chem. 78, 3326-3334 (2006).

67 Lee, C.- Y., Gong, P., Harbers, G. M., Grainger, D. W., Castner, D. G. \& Gamble, L. J. Surface coverage and structure of mixed DNA/alkylthiol monolayers on gold: characterization by XPS, NEXAFS, and fluorescence intensity measurements. Anal. Chem. 78, 3316-3325 (2006)

68 Levicky, R., Herne, T. M., Tarlov, M. J. \& Satija, S. K. Using self-assembly to control the structure of DNA monolayers on gold: a neutron reflectivity study. J. Am. Chem. Soc. 120, 9787-9792 (1998).

69 Boozer, C., Chen, S. \& Jiang, S. Controlling DNA orientation on mixed ssDNA/OEG SAMs. Langmuir 22, 4694-4698 (2006).

70 Herne, T. M. \& Tarlov, M. J. J. Characterization of DNA probes immobilized on gold surfaces. Am. Chem. Soc. 119, 8916-8920 (1997).

71 Peterlinz, K. A., Georgiadis, R. M., Herne, T. M. \& Tarlov, M. J. Observation of hybridization and dehybridization of thiol-tethered DNA using two-color surface plasmon resonance spectroscopy. J. Am. Chem. Soc. 119, 3401-3402 (1997).

72 Nagasaki, Y. Polyethylene glycol-b-polyamine stabilized bionanoparticles for nanodiagnostics and nanotherapy. Chem. Lett. 37, 564-569 (2008).

73 Yoshimoto, K., Matsumoto, S., Asakawa, R., Uchida, K. \& Nagasaki, Y. Immobilization and hybridization behavior of DNA on poly(ethylene glycol)-block-poly[2-(N,Ndimethylamino) ethyl methacrylate]-modified gold surfaces. Chem. Lett. 36, 1444-1445 (2007).

74 Oishi, M., Nakaogami, J., Ishii, T. \& Nagasaki, Y. Smart PEGylated gold nanoparticles for the cytoplasmic delivery of siRNA to induce enhanced gene silencing. Chem. Lett. 35, 1046-1047 (2006).

75 Xiaofei, Y., Fabregat, D., Yoshimoto, K. \& Nagasaki, Y. Efficient inhibition of interfacial nonspecific interaction to create practically utilizable high ferritin-response immunolatex. Anal. Chem. 81, 10097-10105 (2009).
76 Kubota, M., Yoshimoto, K., Xiaofei, Y. \& Nagasaki, Y. Improvement of the thermal stability of streptavidin immobilized on magnetic beads by the construction of a mixed poly(ethylene glycol) tethered-chain layer. Polymer J. 43, 493-496 (2011).

77 Sakata, T., Maruyama, S., Ueda, A., Otsuka, H. \& Miyahara, Y. Stable immobilization of an oligonucleotide probe on a gold substrate using tripodal thiol derivatives. Langmuir 23, 2269-2272 (2007).

78 Bearinger, J. P., Terrettaz, S., Michel, R., Tirelli, N., Vogel, H., Textor, M. \& Hubbell, J. A. Chemisorbed poly(propylene sulphide)-based copolymers resist biomolecular interactions. Nat. Mater. 2, 259-264 (2003).

79 Feller, L. M., Cerritelli, S., Textor, M., Hubbell, J. A. \& Tosatti, S. G. P. Influence of poly(propylene sulfide-block-ethylene glycol) di- and triblock copolymer architecture on the formation of molecular adlayers on gold surfaces and their effect on protein resistance: a candidate for surface modification in biosensor research. Macromolecules 38, 10503-10510 (2005).

80 Yoshimoto, K., Nozawa, M., Matsumoto, S., Echigo, T., Nemoto, S., Hatta, T. \& Nagasaki, Y. Studies on the adsorption property and structure of polyamine-ended poly(ethylene glycol) derivatives on a gold surface by surface plasmon resonance and angle-resolved X-ray photoelectron spectroscopy. Langmuir 25, 12243-12249 (2009).

81 Konradi, R., Pidhatika, B., Mhlebach, A. \& Textor, M. Poly-2-methyl-2-oxazoline: a peptide-like polymer for protein-repellent surfaces. Langmuir 24, 613-616 (2008).

82 Lee, S. \& Spencer, N. D. Adsorption properties of poly(L-lysine)-graft-poly(ethylene glycol) (PLL-g-PEG) at a hydrophobic interface: influence of tribological stress, $\mathrm{pH}$, salt concentration, and polymer molecular weight. Langmuir 24, 9479-9488 (2008).

83 Nagasaki, Y., Yasugi, K., Kurokawa, K. \& lijima, M. Thermal- and dispersion-stable lipaseinstalled gold colloid: PEGylation of enzyme-installed gold colloid. Colloid Polymer Sci. 285, 563-567 (2007).

84 Xiaofei, Y., Iijima, M., Oishi, M. \& Nagasaki, Y. Structure and activity assay of nanozymes prepared by the coimmobilization of practically useful enzymes and hydrophilic block copolymers on gold nanoparticles. Langmuir 24, 6903-6909 (2008).

85 Yoshimoto, K., Hirase, T., Nemoto, S., Hatta, T. \& Nagasaki, Y. Facile construction of sulfanyl-terminated poly(ethylene glycol)-brushed layer on a gold surface for protein immobilization by the combined use of sulfanyl-ended telechelic and semitelechelic poly(ethylene glycol)s. Langmuir 24, 9623-9629 (2008). 